\title{
Proportional Two Integral (P2I) Observer synthesis for Single Track Vehicle
}

\author{
Chabane Chenane, Dalil Ichalal, Hichem Arioui and Said Mammar
}

\begin{abstract}
This paper deals with state estimation of Powered Two Wheeled (PTW) vehicle and robust reconstruction of related unknown inputs. For this purpose, we consider a Proportional two Integral (P2I) observer. Based on LMI technique, we recover vehicle states and part of its dynamics (rider action) within steering angle disturbances and system uncertainties.

To prove the effectiveness and the robustness of the proposed observer, two different scenarios are considered: first we observe the system with disturbances without system's uncertainties. The second case is more general as the observer synthesis is conducted in the presence of steering angle disturbances/system's uncertainties. Both the state and the rider action, on the handlebar, are recovered in the two cases.
\end{abstract}

\section{INTRODUCTION}

In recent years, the use of powered two wheeled (PTW) vehicles is constantly growing, upsetting driving practices and road traffic. Unfortunately, this change is also followed by an increased risk of accident (20 times higher when driving a car). The statistics confirm this observation and riders are considered as the most vulnerable road users. In 2010, the French Agency of Road Safety made a finding of around 1000 deaths (25\% of traffic fatalities), while the traffic volumes of motorcycles does not exceed $1 \%$, [14]. Several reaserch programs are launched to answer this issue and to find solutions in term of preventive and / or active safety, [1], [3].

The success of these research programs or proposed safety systems depends on the knowledge of: 1) the dynamics of vehicle, including motorcycles, and 2) the evolution of its states (observation/estimation). Regarding the first point, several studies were carried out in order to understand the motorcycle dynamics [18], [7], [9], stability analysis (eigenmodes) of PTW [2], optimal and safe trajectories [4] and the proposal of risk functions [19], [8] to detect borderline cases of loss of adhesion or equilibrium. These research are very few sustainable if they are not backed by a system (sensors or observation technique) for estimating the dynamic states of the bike.

The measurement, by sensors, of all the dynamic states of the bike is not conceivable for two reasons: 1) instrumentation can be very expensive and leading inevitably to expensive new bikes, and 2) according to used technologies, the measurement noise can seriously compromise the future safety systems. Thus, we propose to use the observation techniques to overcome the previous shortcomings. In this

All the authors are with the IBISC Laboratory, Université d'Evry Val d'Essonne, 40, rue de Pelvoux, 91020 Evry cedex \{chabane. chenane\} dibisc.univ-evry.fr context, including all methodologies (Luenberger observer, Takagi-Seguno based observer, Extended Kalman filter), very few studies exist [10], [20]. The present paper proposes a robust proportional two integral approach, [12], [11], [13], [17], helping in states observation of linear motorcycle model and the reconstruction of rider's action (couple or steering angle) under disturbances and system's uncertainties. Disturbances and the rider action are assumed to be almost affine. This assumption is quite realistic according to common variation of roads profile. An $H_{\infty}$ performance index is included during the design in order to attenuate the effect of the second derivative of the Unknown Input (UI) on the estimation error.

This paper is organized as follows: section II is dedicated to the motorcycle model description. Sections III and IV present the robust estimation of the motorcycle states and UI reconstruction. Finally, simulation results and conclusions are given.

\section{DyNAMiC MODEl OF MotorCyClE}

The single track vehicle is more difficult to study than the automobile. Stability of motion is an important issue and should be studied to properly handle the vehicle. In this section, the motorcycle dynamics considered here concerns only the lateral motion (motorcycle and rider body are assumed to be a single rigid body) and the longitudinal speed $v_{x}$ is constant. The contribution of steering and twisting dynamics are neglected (see [20], [15] for details and other assumptions). Last assumptions concern small angles approximation adopted to obtain a linear dynamic model and roll axis is merged the road's plane. Here, the steer angle $\delta$ is the model input.

In figure 1, the motorcycle has been depicted while it moves at a roll angle $\phi$ in the mainframe. The represented motorcycle linearized model can be rewritten as follows:

$$
\begin{aligned}
m\left(\dot{v}_{y}+\dot{\psi} v_{x}\right) & =F_{y f}+F_{y r} \\
I_{z} \ddot{\psi} & =a F_{y f}-b F_{y r} \\
-m h\left(\dot{v}_{y}+\dot{\psi} v_{x}\right) & \\
+I_{x x} \ddot{\phi} & =m g h \phi
\end{aligned}
$$

with :

$$
\begin{gathered}
F_{y f}=C_{f}\left(\delta-\frac{v_{y}-a \dot{\psi}}{v_{x}}\right)+C_{f c} \phi \\
F_{y r}=-C_{r}\left(\frac{v_{y}-b \dot{\psi}}{v_{x}}\right)+C_{r c} \phi
\end{gathered}
$$

where $v_{y}$ denotes the lateral velocity of center of gravity, $\dot{\psi}$ is the yaw angular velocity, $\phi$ and derivatives are the angle, 


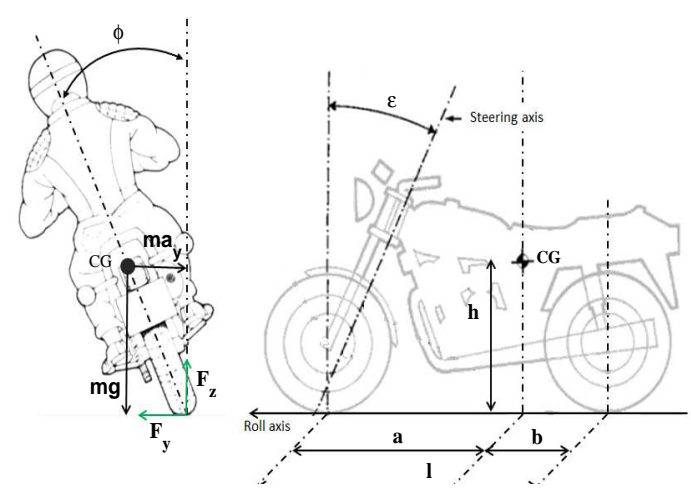

Fig. 1. Motorcycle model configuration

velocity and acceleration of the roll motion. $v_{x}$ represents the longitudinal speed. $F_{y f}$ and $F_{y r}$ are respectively the lateral forces applied by the road on front and rear wheels in the linear region.

$m$ is the sum of total masses of mainframe, front upper frame, front sub-frame and upper body of the rider. $I_{i}$ are the corresponding moment of inertia. $h$ is the distance between center of gravity and the roll axis. $C_{i j}$ are stiffness constants with respect to rear $(i=r)$ and front $(i=f)$ wheels.

One can rewrite the previous dynamic model as linear model state space equation as:

$$
\begin{aligned}
& \dot{x}(t)=A_{1} x(t)+B u(t)+B_{1} F_{h}(t)+B_{2} \omega(t) \\
& y(t)=C x
\end{aligned}
$$

where :

$x(t)$ is the state vector given by $\left[\begin{array}{llll}\dot{\psi} & \dot{\phi} & v_{y} & \phi\end{array}\right]^{T} . F_{h}(t)$ represents the steer angle input $\delta(t), \omega(t)$ is the unknown disturbance input which can model the steering angle disturbance, and $u(t)$ is the feedback control. To stabilize the system, we apply the following control input $u(t)=-K x(t)$, where $K$ is a control gain synthesized using a specific pole placement. Then we write (4) as:

$$
\begin{aligned}
\dot{x}(t) & =\left(A_{1}-B K\right) x(t)+B_{1} F_{h}(t)+B_{2} \omega(t) \\
& =A x(t)+B_{1} F_{h}(t)+B_{2} \omega(t) \\
y(t) & =C x
\end{aligned}
$$

\section{P2I ObSERVER DESIGN UNDER DisturbanCES}

Proportional-Integral (PI) observer is an extension of the Luenberger observer. The presence of the integral of the estimation error makes it effective in estimating the system perturbations and input disturbances. The PI observer can be extended for the estimation of non constant UI faults by the use of Multiple Integral loops (PMI). In fact, any unknown input with zero $k^{\text {th }}$ derivative can be estimated [12].

In the following, we aim to robustly estimate the environmental disturbances which are considered as an unknown input (fault signal). Here, we consider that the second derivative $F_{h}^{(2)}(t)$ of the $F_{h}(t)$ is bounded. The previous system (6) is augmented with $\dot{\xi}_{1}=F_{h}^{(2)}(t), \dot{\xi}_{2}=\xi_{1}=F_{h}^{(1)}(t)$, which leads to the augmented system described by state vector, $\bar{x}=\left[\begin{array}{lll}x^{T} & \xi_{1} & \xi_{2}\end{array}\right]^{T}$. The new state space system is given by:

$$
\begin{aligned}
\dot{\bar{x}}(t) & =\bar{A} \bar{x}(t)+\bar{B} \bar{\omega}(t) \\
y(t) & =\bar{C} \bar{x}(t)
\end{aligned}
$$

with

$$
\begin{gathered}
\bar{A}=\left[\begin{array}{ccc}
A & \mathbf{0} & B_{1} \\
\mathbf{0} & 0 & 0 \\
\mathbf{0} & 1 & 0
\end{array}\right], \bar{B}_{1}=\left[\begin{array}{l}
0 \\
1 \\
0
\end{array}\right], \bar{B}_{2}=\left[\begin{array}{c}
B_{2} \\
0 \\
0
\end{array}\right] \\
\bar{B}=\left[\begin{array}{ll}
\bar{B}_{1} & \bar{B}_{2}
\end{array}\right], \bar{\omega}(t)=\left[\begin{array}{l}
\chi(t) \\
\omega(t)
\end{array}\right] \text { and } \bar{C}=\left[\begin{array}{lll}
C & \mathbf{0} & \mathbf{0}
\end{array}\right]
\end{gathered}
$$

where $\chi(t)=F_{h}^{(2)}(t)$.

Note that zeros in bold are zero matrices with appropriate dimensions. The necessary requirement for the P2I observer design is the full observability of the augmented system $(\bar{A}, \bar{C})$.

Let us consider now the following state-space observer:

$$
\dot{\hat{x}}(t)=\bar{A} \hat{\bar{x}}(t)+\bar{L}(y(t)-\bar{C} \hat{\bar{x}}(t))
$$

the vector $\bar{L}$ is the gain to be designed.

Lets $e(t)=\bar{x}-\hat{\bar{x}}$ be the estimation error. Its time derivative is given by:

$$
\begin{aligned}
\dot{e}(t) & =\dot{\bar{x}}(t)-\dot{\hat{\bar{x}}}(t) \\
& =(\bar{A}-\bar{L} \bar{C}) e(t)+\bar{B} \bar{\omega}(t)
\end{aligned}
$$

The considered Lyapunov function is:

$$
V(t)=e^{T}(t) P e(t)
$$

where $P$ is a symmetric positive definite matrix. The derivative of the Lyapunov function is given by:

$\dot{V}=e^{T}\left[P(\bar{A}-\bar{L} \bar{C})+(\bar{A}-\bar{L} \bar{C})^{T} P\right] e+\bar{\omega}^{T} \bar{B}^{T} P e+e^{T} P \bar{B} \bar{\omega}$

The observer gain $\bar{L}$ is computed in order to stabilize the system generating the state estimation error and also to attenuate the effect of the Unknown Input, $\bar{\omega}(t)$, on the estimation error, namely $\left\|T_{e \bar{\omega}}\right\|_{\infty}<\gamma, \gamma>0$, where $T_{e \bar{\omega}}$ represents the transfer from $\bar{\omega}(t)$ to the error $e(t)$.

Let us define the $L_{2}$-gain of the system as the quantity:

$$
\sup \frac{\|e\|_{2}}{\|\bar{\omega}\|_{2}}, \quad\|\bar{\omega}\|_{2} \neq 0
$$

where the $L_{2}$-norm of $e(t)$ and $\bar{\omega}$ are defined by:

$$
\begin{aligned}
\|e(t)\|_{2} & =\left(\int_{0}^{\infty} e(t)^{T} e(t) d t\right)^{1 / 2} \\
\|\bar{\omega}(t)\|_{2} & =\left(\left(\int_{0}^{\infty} \bar{\omega}(t)^{T} \bar{\omega}(t) d t\right)\right)^{1 / 2}
\end{aligned}
$$

Then, if the inequality

$$
\dot{V}(t)+e^{T} e-\gamma^{2} \bar{\omega}^{T} \bar{\omega}<0
$$


holds, the state estimation error dynamics is stable and the transfer from $\bar{\omega}(t)$ to $e(t)$ is bounded by $\gamma$. By replacing (14) in (18) one obtains:

$$
\begin{aligned}
& e^{T}\left[P(\bar{A}-\bar{L} \bar{C})+(\bar{A}-\bar{L} \bar{C})^{T} P+I\right] e \\
+\quad & \bar{\omega}^{T} \bar{B}^{T} P e+e^{T} P \bar{B} \bar{\omega}-\gamma^{2} \bar{\omega}^{T} \bar{\omega}<0
\end{aligned}
$$

Since the final objective is to derive LMI conditions, let us consider the change of variable $\bar{L}=\bar{P}^{-1} \bar{Z}$. The inequality (19) is equivalent to

$$
\begin{aligned}
& e^{T}\left[\bar{A}^{T} \bar{P}+\bar{P} \bar{A}-\bar{C}^{T} \bar{Z}^{T}-\bar{Z} \bar{C}+I\right] e \\
+\quad & \bar{\omega}^{T} \bar{B}^{T} P e+e^{T} P \bar{B} \bar{\omega}-\gamma^{2} \bar{\omega}^{T} \bar{\omega}<0
\end{aligned}
$$

In matrix formulation, (20) is equivalent to

$$
\left(\begin{array}{c}
e \\
\bar{\omega}
\end{array}\right)^{T}\left(\begin{array}{cc}
\bar{A}^{T} \bar{P}+\bar{P} \bar{A}-\bar{C}^{T} \bar{Z}^{T}-\bar{Z} \bar{C}+I & \bar{P} \bar{B} \\
\bar{B}^{T} \bar{P} & -\gamma^{2} I
\end{array}\right)\left(\begin{array}{c}
e \\
\bar{\omega}
\end{array}\right)<0
$$

The quadratic form (21) is negative definite if and only if the linear matrix inequality

$$
\left(\begin{array}{cc}
\bar{A}^{T} \bar{P}+\bar{P} \bar{A}-\bar{C}^{T} \bar{Z}^{T}-\bar{Z} \bar{C}+I & \bar{P} \bar{B} \\
\bar{B}^{T} \bar{P} & -\gamma^{2} I
\end{array}\right)<0
$$

holds. Finally, given a scalar $\gamma$, if there exists a symmetric and positive definite matrix $P$ and a matrix $\bar{Z}$ such that the LMI (22) is satisfied, then the system generating the state estimation error is stable and the transfer from $\bar{\omega}(t)$ to $e(t)$ is bounded by the $L_{2}$ gain $\gamma$. Furthermore, in order to enhance the performances of the observer, it is possible to minimize the transfer gain $\gamma$ subject to LMI constraints. The following optimization problem is then stated

$$
\left(\begin{array}{cc}
\bar{A}^{T} \bar{P}+\bar{P} \bar{A}-\bar{C}^{T} \bar{Z}^{T}-\bar{Z} \bar{C}+I & \bar{P} \bar{B} \\
\bar{B}^{T} \bar{P} & -\bar{\gamma} I
\end{array}\right)<0
$$

by choosing $\gamma$ as a variable and using the change of variables $\bar{\gamma}=\gamma^{2}$. After solving this optimization problem, the gains of the observer are obtained by $L=P^{-1} \bar{Z}$ and the attenuation gain is given by $\gamma=\sqrt{\bar{\gamma}}$.

In the following section, we will consider the uncertainty of modeling and the disturbances impact as two separate problems.

\section{P2I ObSERVER DESIGN UNDER DisturbancES AND UNCERTAIN SYSTEM PARAMETERS}

In this part, the system is considered uncertain and subjected to parameters variation on the matrices $A$ and $B_{1}$. In similar way, as in the first part, the augmented system is obtained. The parameters variations are modeled as timevarying uncertainties $\Delta \bar{A}(t)$. The augmented system is written as follows:

$$
\begin{aligned}
\dot{\bar{x}}(t) & =(\bar{A}+\Delta \bar{A}(t)) \bar{x}(t)+\bar{B} \bar{\omega}(t) \\
y(t) & =\bar{C} \bar{x}(t)
\end{aligned}
$$

Considering the same P2I observer of equation (10), the time derivative of the estimation error is now given by:

$$
\begin{aligned}
\dot{e}(t) & =\dot{\bar{x}}(t)-\dot{\bar{x}}(t) \\
& =(\bar{A}-\bar{L} \bar{C}) e(t)+\bar{B} \bar{\omega}(t)+\Delta \bar{A} \bar{x}(t) \\
& =(\bar{A}-\bar{L} \bar{C}) e(t)+\bar{B} \bar{\omega}(t)+\Delta \tilde{A} x(t)+\Delta \tilde{B} \bar{F}_{h}
\end{aligned}
$$

where :

$$
\Delta \tilde{A}=\left(\begin{array}{c}
\Delta A \\
\mathbf{0}
\end{array}\right), \Delta \tilde{B}=\left(\begin{array}{cc}
\mathbf{0} & \Delta B_{1} \\
0 & 0 \\
1 & 0
\end{array}\right), \bar{F}_{h}=\left(\begin{array}{c}
\dot{F}_{h} \\
F_{h}
\end{array}\right)
$$

Then we can write:

$$
\dot{\bar{x}}(t)=(A+\Delta A(t)) x(t)+\left(B_{1}+\Delta B_{1}\right) F_{h}+B_{2} \omega
$$

Let us define the augmented vector $\tilde{x}=\left[\begin{array}{ll}e^{T} & x^{T}\end{array}\right]^{T}$, we can obtain the following system:

$$
\begin{gathered}
\dot{\tilde{x}}=\mathscr{A} \tilde{x}+\mathscr{B} \tilde{\omega} \\
z=H \tilde{x} \\
\mathscr{A}=\left(\begin{array}{cc}
\Phi & \Delta \tilde{A} \\
\mathbf{0} & A+\Delta A
\end{array}\right), \mathscr{B}=\left(\begin{array}{cc}
\bar{B} & \Delta \tilde{B} \\
\Lambda_{1} & \Lambda_{2}
\end{array}\right) \\
\Lambda_{1}=\left[\begin{array}{ll}
\mathbf{0} & B_{2}
\end{array}\right], \quad \Lambda_{2}=\left[\begin{array}{ll}
\mathbf{0} & B_{1}+\Delta B_{1}
\end{array}\right] \\
\Phi=(\bar{A}-\bar{L} \bar{C}), \quad \tilde{\omega}=\left[\begin{array}{ll}
\bar{\omega}, & \bar{F}_{h}
\end{array}\right]^{T}, \quad H=\left[\begin{array}{ll}
I & 0
\end{array}\right]
\end{gathered}
$$

Let us consider the same Lyapunov function $V(t)=$ $\tilde{x}^{T}(t) P \tilde{x}(t)$. The system (29)-(30) is stable and the gain $L_{2}$ of the transfer from $\tilde{\omega}(t)$ to $z(t)$ is bounded by $\gamma$ if the following condition holds:

$$
\dot{V}(t)+z^{T}(t) z(t)-\gamma^{2} \tilde{\omega}^{T}(t) \tilde{\omega}(t)<0
$$

we obtain:

$$
\left(\begin{array}{c}
\tilde{x} \\
\tilde{\omega}
\end{array}\right)^{T}\left(\begin{array}{cc}
\mathscr{A}^{T} P+P \tilde{A A}+H^{T} H & P \mathscr{B} \\
(P \mathscr{B})^{T} & -\gamma^{2} I
\end{array}\right)\left(\begin{array}{c}
\tilde{x} \\
\tilde{\omega}
\end{array}\right)<0
$$

Let us consider the following particular form of the matrix $P$ :

$$
P=\left(\begin{array}{cc}
P_{1} & \mathbf{0} \\
\mathbf{0} & P_{2}
\end{array}\right)
$$

then after replacing all the matrices, one can obtain:

$$
\left(\begin{array}{c}
e(t) \\
x(t) \\
\bar{\omega}(t) \\
\bar{F}_{h}(t)
\end{array}\right)^{T} \quad \Xi\left(\begin{array}{c}
e(t) \\
x(t) \\
\bar{\omega} \\
\bar{F}_{h}(t)
\end{array}\right)<0
$$

If $(\Xi<0)$ the inequality (31) is hold, where:

$$
\Xi=\left(\begin{array}{cccc}
\Pi & P_{1} \Delta \tilde{A} & P_{1} \bar{B} & P_{1} \Delta \tilde{B} \\
* & \Psi & P_{2} \Lambda_{1} & P 2 \Lambda_{2} \\
* & * & -\gamma^{2} I & 0 \\
* & * & * & -\gamma^{2} I
\end{array}\right)
$$

where $(*)$ represents the symmetric and transposed term and where:

$$
\begin{gathered}
\Pi=\Phi^{T} P_{1}+P_{1} \Phi+I \\
\Psi=A^{T} P_{2}+P_{2} A+P_{2} \Delta A+\Delta A^{T} P_{2}
\end{gathered}
$$




$$
\begin{aligned}
& \Xi=\left[\begin{array}{cccc}
\Pi & \mathbf{0} & P_{1} \bar{B} & \mathbf{0} \\
* & A^{T} P_{2}+P_{2} A & P_{2}\left[\begin{array}{cc}
\mathbf{0} & B_{2}
\end{array}\right] & P_{2}\left[\begin{array}{cc}
\mathbf{0} & B_{1}
\end{array}\right] \\
* & * & -\gamma^{2} I & \mathbf{0} \\
* & * & * & -\gamma^{2} I
\end{array}\right] \\
& +\left[\begin{array}{cccc}
\mathbf{0} & P_{1} \Delta \tilde{A} & \mathbf{0} & P_{1} \Delta \tilde{B} \\
\left(P_{1} \Delta \tilde{A}\right)^{T} & P_{2} \Delta A+\Delta A^{T} P_{2} & \mathbf{0} & P_{2} \Delta \breve{B}_{1} \\
\mathbf{0} & \mathbf{0} & \mathbf{0} & \mathbf{0} \\
\left(P_{1} \Delta \tilde{B}\right)^{T} & \left(P_{2} \Delta \breve{B}_{1}\right)^{T} & \mathbf{0} & \mathbf{0}
\end{array}\right]
\end{aligned}
$$

$\Xi$ is divided into two parts with respect to time dependence in order to derive time independence matrix. The uncertain terms are defined by:

$$
\Delta A(t)=N \Sigma(t) E_{1}, \Delta \tilde{A}=\tilde{N}_{A} \tilde{\Sigma}_{A}(t) \tilde{E}_{A}, \Delta \tilde{B}=\tilde{N}_{B} \tilde{\Sigma}_{B}(t) \tilde{E}_{B}
$$
,$\Delta \breve{B}_{1}=\breve{N} \breve{\Sigma}(t) \breve{E}$. By applying the claim [16], we can say that for $X, Y, F$ real matrices with appropriate dimension verifying $F^{T} F \leq 1$ and for any scalar $\varepsilon>0$, the following inequality holds:

$$
X F^{T} Y^{T}+Y F X^{T} \leq \varepsilon X X^{T}+\varepsilon^{-1} Y Y^{T}
$$

then, the second part of (34) can be bounded as follows:

$$
\begin{array}{r}
{\left[\begin{array}{cccc}
\mathbf{0} & P_{1} \Delta \tilde{A} & \mathbf{0} & P_{1} \Delta \tilde{B} \\
\left(P_{1} \Delta \tilde{A}\right)^{T} & \mathbf{0} & \mathbf{0} & \mathbf{0} \\
\mathbf{0} & \mathbf{0} & \mathbf{0} & \mathbf{0} \\
\left(P_{1} \Delta \tilde{B}\right)^{T} & \mathbf{0} & \mathbf{0} & \mathbf{0}
\end{array}\right]} \\
+\left[\begin{array}{ccccc}
\mathbf{0} & \mathbf{0} & \mathbf{0} & \mathbf{0} \\
\mathbf{0} & P_{2} \Delta A+\Delta A^{T} P_{2} & \mathbf{0} & \mathbf{0} \\
\mathbf{0} & \mathbf{0} & \mathbf{0} & \mathbf{0} \\
\mathbf{0} & \mathbf{0} & \mathbf{0} & \mathbf{0}
\end{array}\right] \\
+\left[\begin{array}{cccc}
\mathbf{0} & \mathbf{0} & \mathbf{0} & \mathbf{0} \\
\mathbf{0} & \mathbf{0} & \mathbf{0} & P_{2} \Delta \breve{B}_{1} \\
\mathbf{0} & \mathbf{0} & \mathbf{0} & \mathbf{0} \\
\mathbf{0} & \left(P_{2} \Delta \breve{B}_{1}\right)^{T} & \mathbf{0} & \mathbf{0}
\end{array}\right]
\end{array}
$$

Using (35), we have

$$
P_{2} \Delta A+\Delta A^{T} P_{2} \leq \varepsilon E_{1}^{T} E_{1}+\varepsilon^{-1} P_{2} N N^{T} P_{2}
$$

then we obtain:

$$
\left[\begin{array}{cccc}
\pi_{1} & \mathbf{0} & \mathbf{0} & \mathbf{0} \\
\mathbf{0} & \pi_{2} & \mathbf{0} & \mathbf{0} \\
\mathbf{0} & \mathbf{0} & \mathbf{0} & \mathbf{0} \\
\mathbf{0} & \mathbf{0} & \mathbf{0} & \pi_{3}
\end{array}\right]
$$

where:

$$
\begin{aligned}
& \pi_{1}=\lambda_{1}{ }^{-1} P_{1} \tilde{N}_{A} \tilde{N}_{A}^{T} P_{1}+\lambda_{2}{ }^{-1} P_{1} \tilde{N}_{B} \tilde{N}_{B}^{T} P_{1} \\
& \pi_{2}=\varepsilon E_{1}{ }^{T} E_{1}+\varepsilon^{-1} P_{2} N N^{T} P_{2}+\lambda_{1}{\tilde{E_{A}}}^{T} \tilde{E}_{A}+\lambda_{3}{ }^{-1} P_{2} \breve{N} \breve{N}^{T} P_{2}
\end{aligned}
$$

$$
\pi_{3}=\lambda_{2}{\tilde{E_{B}}}^{T} \tilde{E}_{B}+\lambda_{3} \breve{E}^{T} \breve{E}
$$

Using the variable transform $\bar{L}=P_{1}^{-1} \bar{Z}$ and the following Schur complement given in the lemma 1:

Lemma 1: [5] Let us consider the matrices $Q=Q^{T}$, $R=R^{T}$ and $S$ with appropriate dimensions. The following inequalities are equivalent:

$$
\text { - }\left(\begin{array}{cc}
Q & S \\
S^{T} & R
\end{array}\right)<0
$$

- $R<0, Q-S R^{-1} S^{T}<0$

we can write the above nonlinear matrix inequality $\Xi<0$ into a linear matrix inequality:

$\left[\begin{array}{cccccccc}\Omega_{1} & \mathbf{0} & P_{1} \bar{B} & \mathbf{0} & P_{1} \tilde{N}_{A} & P_{1} \tilde{N}_{B} & \mathbf{0} & \mathbf{0} \\ * & \Omega_{2} & P_{2} \eta_{1} & P_{2} \eta_{2} & \mathbf{0} & \mathbf{0} & P_{2} N & P_{2} \breve{N} \\ * & * & -\gamma^{2} I & \mathbf{0} & \mathbf{0} & \mathbf{0} & \mathbf{0} & \mathbf{0} \\ * & * & * & -\gamma^{2} I+\pi_{3} & \mathbf{0} & \mathbf{0} & \mathbf{0} & \mathbf{0} \\ * & * & * & * & -\lambda_{1} I & \mathbf{0} & \mathbf{0} & \mathbf{0} \\ * & * & * & * & * & -\lambda_{2} I & \mathbf{0} & \mathbf{0} \\ * & * & * & * & * & * & -\varepsilon I & \mathbf{0} \\ * & * & * & * & * & * & * & -\lambda_{3} I\end{array}\right]<0$

$$
\begin{aligned}
\Omega_{1} & =P_{1} \bar{A}+\bar{A}^{T} P_{1}-\bar{Z} \bar{C}-\bar{C}^{T} \bar{Z}^{T}+I \\
\Omega_{2} & =A^{T} P_{2}+P_{2} A+\varepsilon^{-1} E_{1}^{T} E_{1}+\lambda_{1}{\tilde{E_{A}}}^{T} \tilde{E}_{A} \\
\eta_{1} & =\left[\begin{array}{ll}
\mathbf{0} & B_{2}
\end{array}\right], \quad \eta_{2}=\left[\begin{array}{ll}
\mathbf{0} & B_{1}
\end{array}\right]
\end{aligned}
$$

In a similar way, as in the first part, it is possible to enhance the performances of the observer by minimizing the parameter $\gamma$. The change of variable $\bar{\gamma}=\gamma^{2}$ is then used. An optimization problem is then obtained by minimizing $\gamma$ under LMI constraint (42) after changing $\gamma^{2}$ by $\bar{\gamma}$.

\section{Simulation Results}

The model considered here is that of a two-wheeled vehicle with a single body, where $F_{h}(t)$ is the steering angle. All parameters are given in the appendix. The objective is to estimate the state vector and the steering angle of the motorcycle. For that purpose, three simulations are proposed in order to illustrate the two approaches. The first one uses the first method taking into account the external disturbances $\omega(t)$, which is considered as a centered random signal (can be considered, in practice, as the disruption resulting from road irregularities or aerodynamic forces, etc.) with maximal amplitude 2 and without taking into account the parameter uncertainties, since the second simulation deals with the problem of observer design with taking into account the uncertainties of the model and the effect of the perturbations $\omega(t)$. It is possible to enhance the performances of the observer by choosing an LMI region in order to limit the imaginary part of the dynamics matrix of the observer which limits the effect of the oscillatory phenomenon in the transit phase. To do that the LMI region $S$ defined by $S$ defined by $S=\{z \in C|\operatorname{Re}(z)<-a| z \mid,<R\}$, is chosen, which is an intersection between the left plan defined by the $\operatorname{Re}(z)<-a$ and the disc with center $(0,0)$ and radius $R$. So, the problem is now to solve simultaneously the proposed optimization problems and the LMI constraints, corresponding to the LMI region, given as follows (for more details see [6])

$$
\begin{gathered}
\bar{A}^{T} P+P \bar{A}-\bar{Z} C-C^{T} \bar{Z}^{T}+2 a P<0 \\
{\left[\begin{array}{cc}
-R P & P \bar{A}-\bar{Z} C \\
\bar{A}^{T} P-C^{T} \bar{Z}^{T} & -R P
\end{array}\right]<0}
\end{gathered}
$$

In all the simulations $a=12$ and $R=15$. 


\section{A. State and unknown input estimation}

By solving the optimization problem minimizing $\bar{\gamma}$ subject to the LMI constraint (23), the following gain of the observer is obtained:

$$
L=\left(\begin{array}{cc}
-27.7916 & 38.5655 \\
-42.6005 & 153.5974 \\
-137.8306 & 341.5682 \\
-26.0097 & 69.3984 \\
-19.7071 & 65.3497 \\
-8.2148 & 24.7726
\end{array}\right)
$$

The obtained attenuation level is $\gamma=0.6665$. The simulation results concerning the state estimation is depicted in the figure 2. The results concerning the unknown input (steering angle) is given in the figure 3 . We can note that we have an acceptable results and a good attenuation level of the external disturbances.



Fig. 2. Actual states (blue line) and estimation (dashed red line)
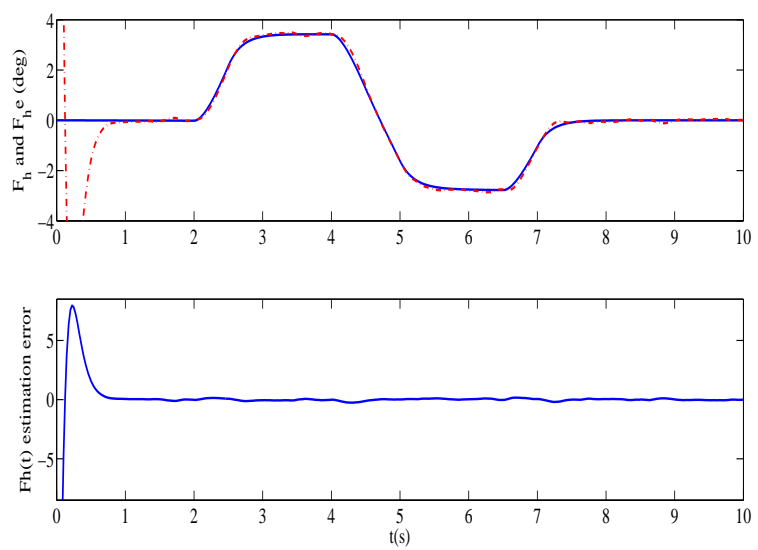

Fig. 3. Steering angle and its estimation (top) and estimation error (bottom)

\section{B. Robust state and unknown input estimation}

In this sub-section, the parameter are assumed to be uncertain (can be considered, in practice, as the unmodeled dynamics or error in identification parameters or changes in road adhesion coefficient, etc.) and modeled with norm bounded uncertainties given by $\Delta A(t)=N_{A} \Sigma(t) E_{A}$ and $\Delta B_{1}=$ $N_{B} \Sigma(t) E_{B}$ and defined by $N_{A}=0.1 I, E_{A}=0.1 * A, N_{B}=0.1 I$, $E_{B}=0.1 * B_{1}$, and $\Sigma(t)=\cos (t) * I$ where $I$ is the identity matrix with dimension 4 . By solving the optimization problem minimizing $\bar{\gamma}$ subject to the LMI constraint (42), the obtained attenuation level is $\gamma=13.03$.

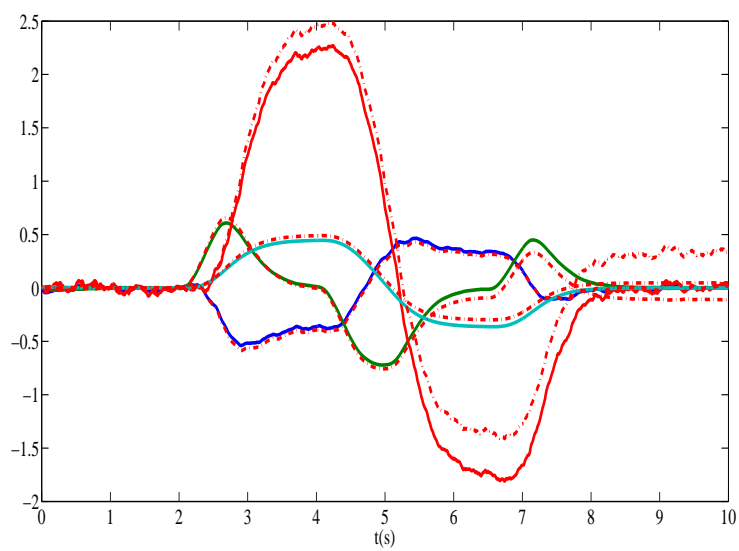

Fig. 4. Nominal system vs uncertain system

In the figure 4 the effect of the uncertainties on the system is illustrated by the states of the nominal system and those of the uncertain one. The states and the unknown input and their estimations are depicted in the figures 5 and 6 .
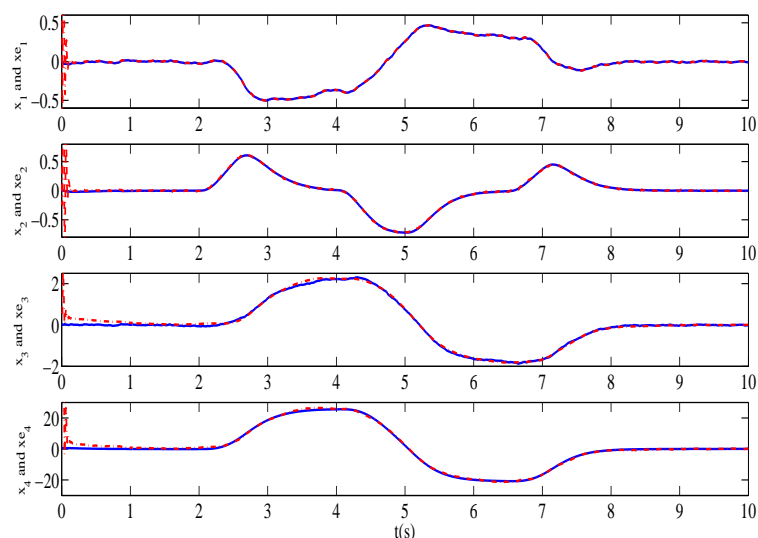

Fig. 5. States and their estimations

As a conclusion, with this matrix $\bar{L}$ we can design the UI observer to estimate the $\hat{\bar{x}}(t)$. We see that the observer is estimating the state vector $\left[\begin{array}{llll}\dot{\psi} & \dot{\phi} & V_{y} & \phi\end{array}\right]^{T}$ and the UI quite efficiently.

\section{CONCLUSION AND FUTURE WORK}

\section{A. Conclusion}

A P2I observer, based on LMI approach, is designed for linear motorcycle model. From results, the proposed 

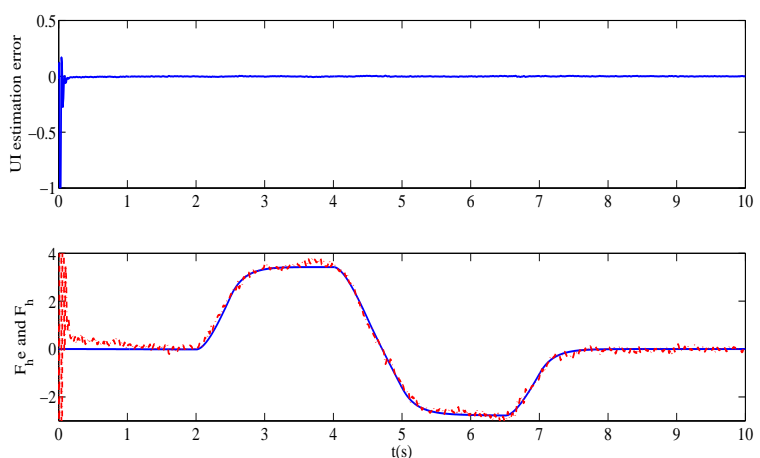

Fig. 6. UI estimation (bottom) and UI estimation error

observer can handle both perturbation (road irregularities and aerodynamic forces) and uncertainties (identification error in motorcycle inertial parameters) robustly. This observation approach is efficient because it allows to reconstruct motorcycle unknown inputs (rider action and/or tire-road forces).

\section{B. Future Works}

In the near future, we plan to consolidate these results with validation on a real benchmark, which is electrical scooter, 7. This motorcycle is almost instrumented with steering angle sensors, inertial unit (linear acceleration and angular velocity), angular speeds of the two wheels and an embedded data acquisition system. Other sensors are planned to be added. This work will be, also, extended for both purposes: 1) take into account more motorcycle dynamics (steering, twisting, longitudinal, etc.) and 2) reconstruction of other inputs as force of tire-road.

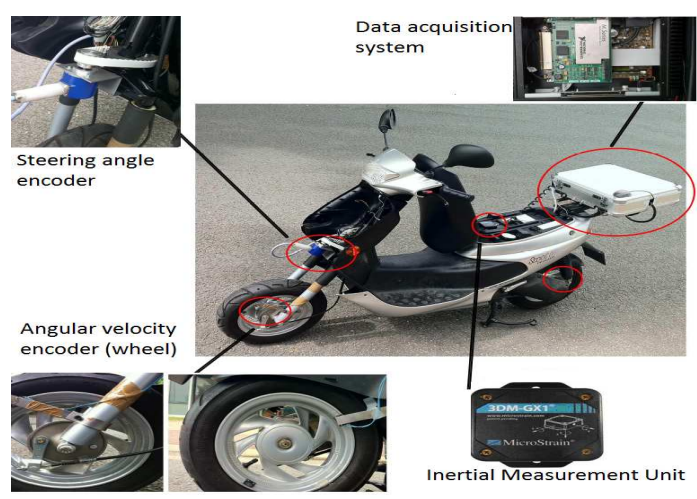

Fig. 7. Sensors implementation on our Motorcycle

\section{APPENDIX}

$$
A_{1}=\left(\begin{array}{cccc}
-39.47 & -2153 & 20.34 & -192.06 \\
3.77 & -10.86 & -2.84 & -12.17 \\
-10.88 & -2.52 & -0.66 & -12.63 \\
0 & 1 & 0 & 0
\end{array}\right)
$$

$$
\begin{aligned}
& B_{1}=\left(\begin{array}{c}
438.40 \\
221.23 \\
51.38 \\
0
\end{array}\right), B_{2}=\left(\begin{array}{l}
0 \\
0 \\
1 \\
0
\end{array}\right), C=\left(\begin{array}{llll}
1 & 0 & 0 & 0 \\
0 & 1 & 0 & 0
\end{array}\right) \\
& m=217.43 \quad h=0.6140 \quad I_{z}=20.95 \\
& I_{x}=31.01 \quad g=9.81 \quad a=0.822 \\
& b=0.478 \quad v_{x}=10 \quad C_{f}=11173.37 \\
& C_{r}=15830.4
\end{aligned}
$$

\section{REFERENCES}

[1] B. Amans and M. Moutreuil. Rider project . research on accidents involving powered two-wheelers. In Final Report $n$ RIDER20050310, French National Agency of Reaserch., France, 2005.

[2] Karl J. Åström, Richard E. Klein, and Anders Lennartsson. Bicycle dynamics and control. IEEE Control Systems Magazine, 25(4):26-47, August 2005.

[3] Evangelos Bekiaris. Saferider european project. In Final Report $n$ RIDER200503-10, E-U, 2008-2010.

[4] S. Bobbo, V. Cossalter, M. Massaro, and M. Peretto. Application of the optimal maneuver method for enhancing racing motorcycle performance. SAE International Journal of Passenger Cars - Mechanical Systems, 1(1):1311-1318, April 2009.

[5] S. Boyd, L. El Ghaoui, E. Feron, and V. Balakrishnan. Linear Matrix Inequalities in System and Control Theory. SIAM ed., 1994.

[6] M. Chilali and P. Gahinet. H-infinity design with pole placement constraints : an LMI approach. IEEE Transactions on Automatic Control, 41(3):358-367, 1996.

[7] V. Cossalter. Motorcycle Dynamics. Lulu.com, 2006.

[8] S. Evangelou. Influence of road camber on motorcycle stability. volume 75, page 231, 2008.

[9] S. Hima, L. Nehaoua, N. Seguy, and H. Arioui. Suitable two wheeled vehicle dynamics synthesis for interactive motorcycle simulator. In $17^{\text {th }}$ IFAC World Congress 2008, Seoul, South of Korea, pages 96101, 2008.

[10] D. Ichalal, H. Arioui, and S. Mammar. Observer design for two-wheeled vehicle: A takagi-sugeno approach with unmeasurable premise variables. In 19th Mediterranean Conference on Control \& Automation (MED'11), Corfu, Greece, June 20-23 2011.

[11] D. Ichalal, B. Marx, J. Ragot, and D. Maquin. Simultaneous state and unknown inputs estimation with PI and PMI observers for Takagi-Sugeno model with unmeasurable premise variables. In 17th Mediterranean Conference on Control and Automation, MED'09, Thessaloniki, Greece, June 24-26 2009.

[12] D. Koenig. Unknown input proportional multiple-integral observer design for linear descriptor systems: application to state and fault estimation. IEEE Transactions on Automatic Control, 50(2):212-217, 2005.

[13] S.P. Linder, B. Shafai, and M. Saif. Estimating and accommodating unknown actuator faults with pi observers. Proceedings of the Conference on Control Applications, Trieste, Italy, pages 461-465, 1998.

[14] French Interministerial National Observatory of Road Safety. characteristics and causes of road accidents, 2010. France, 2010.

[15] H.B. Pacejka and Society of Automotive Engineers. Tire and vehicle dynamics. SAE-R. SAE International, 2006.

[16] I.R. Petersen. A stabilization algorithm for a class of uncertain linear system. Systems and Control Letters, 1(8):351-357, 1987.

[17] B. Shafai, C. Pi, and S. Nork. Simultaneous disturbance attenuation and fault detection using proportional integral observers. Proceedings of American Control Conference (ACC), Anchorage, AK, pages 16431649, 2002.

[18] R.S. Sharp, S. Evangelou, and D.J.N. Limebeer. Advances in the modelling of motorcycle dynamics. Multibody System Dynamics, 12:251-283, 2004.

[19] H. Slimi, H. Arioui, L. Nouvelire, and S. Mammar. Motorcycle speed profile in cornering situation. In American Control Conference (ACC'10), Baltimore, Maryland, USA, 30 June - 02 July, pages 11721177, 2010.

[20] B.A.M. van Daal. Design and automatic tuning of a motorcycle state estimator. PhD thesis, TNO Science \& Industry, Eindhoven University of Technology, Eindhoven, Nederland, 2009. 